\title{
Extraordinary Toughening and Strengthening Effect in Polymer Nanocomposites using Lignin-Based Fillers Synthesized by ATRP
}

Tejank Shah ${ }^{1}$, Chetali Gupta ${ }^{1}$, Rachel L. Ferebee ${ }^{1}$, Michael R. Bockstaller ${ }^{1, *}$, Newell R. Washburn ${ }^{2,3, *}$

${ }^{1}$ Department of Materials Science \& Engineering, Carnegie Mellon University, 5000 Forbes Ave., Pittsburgh, PA 15213

${ }^{2}$ Department of Chemistry, Carnegie Mellon University, 4400 Fifth Ave., Pittsburgh, PA 15213

${ }^{3}$ Department of Biomedical Engineering, Carnegie Mellon University, 4400 Fifth Ave., Pittsburgh, PA 15213

washburn@andrew.cmu.edu, bockstaller@cmu.edu

\begin{abstract}


Understanding of the governing parameters that control the interaction of bio-sourced fillers with synthetic polymer materials is a long-standing challenge for their exploitation as a platform for material engineering. For the case of graft-lignin embedded in poly(methyl methacrylate) (PMMA) it is demonstrated that tethering of polymeric chains with appropriate chain length to the surface of lignin-fillers dramatically increases the mechanical properties of PMMA/lignin composites, suggesting the PMMA grafts significantly enhanced filler-matrix interactions. Most metrics were maximized at $1 \%$ loading, with a 3-fold increase in yield stress, a 4-fold increase in tensile strength, and a 7-fold increase in toughness, with a combination of properties that compare favorably to high-performance engineering polymers and polymer nanocomposites based on inorganic nanoparticles. The versatility of the surface-initiated controlled radical polymerization used for polymer graft modification suggests that the approach should be broadly applicable to a wide range of commodity and engineering polymers.

Keywords: lignin; ATRP; nanocomposite 


\section{Introduction}

Research in polymer nanocomposites has been motivated by the opportunities to enhance or augment the physical properties of polymeric materials. Biomass-derived fillers, including cellulose nanofibers or lignin, have attracted particular interest in the context of 'sustainable materials engineering' as a means towards more benign (and low-energy) polymer materials. However, although the concept of reducing the environmental footprint of synthetic polymers by dilution with bio-sourced fillers appears to be straightforward, the loss of desirable physical properties, such as mechanical strength or optical clarity, that is often observed in biomass-based polymer composites limits the impact of the 'nanocomposite approach' for sustainable material engineering. The effect of filler addition on the physical properties of polymers sensitively depends on the morphology of the composite material and filler-matrix interactions. Aggregation of dispersed particle fillers is generally considered to be detrimental to physical properties of nanocomposites due to embrittlement of the material as well as the loss of optical clarity.(1-4) This presents a particular challenge for biobased fillers - such as lignin - due their inherent chemical incompatibility with most engineering plastics. $(5,6)$ For example, blends of hydroxypropyl lignin in PMMA were found to exhibit a strain-to-fracture of less than $2 \%$ (about half of the value typically observed for pristine PMMA at moderate strain rates) - the embrittlement was attributed to weak polymer-filler interactions and the formation of micron-scale lignin aggregates.(7) A large number of studies involving both bio-sourced as well as inorganic particle fillers have corroborated the particular relevance 
of filler-matrix interactions as governing parameter that determines the physical properties of polymer nanocomposite materials.(8)

A substantial amount of research - mostly on the phase behavior of inorganic particle-in-polymer dispersions - has established the sensitive relation between miscibility and architecture of polymer-tethered particle fillers (here the term architecture is used to describe the relevant structural parameters of polymer-tethered particle systems such as particle size, density as well as degree of polymerization and dispersity of grafted chains). For example, in the case of 'neutral' polymer grafts, in which the chemical composition of the grafted chains is identical to the matrix, miscibility has been shown to increase with the degree of polymerization of surface-tethered chains.(8-12) Parallel progress in surface-initiated controlled radical polymerization has established a range of synthetic methodologies to facilitate the tethering of polymer chains with precisely controlled degree of polymerization, grafting density and even modality of polymeric tethers. $(8,13,14)$ Recently our group demonstrated that the application of surfaceinitiated atom transfer controlled radical polymerization (SI-ATRP) facilitates the efficient synthesis of polymer tethered kraft lignin with high molecular polymeric tethers.(15) SI-ATRP is a particularly interesting technique in the context of filler modification because of its applicability to a wide range of monomer chemistries, the high level of control over molecular characteristics of tethered chains as well as its proven economic efficacy and scalability. $(8,16,17)$ Grafting of polymeric chains was shown to result in lignin-hybrid particles that could be assembled into 'one-component lignin-hybrid materials' with enhanced cohesive interactions as compared to the pristine lignin and polymer constituents - the latter was attributed to the occurrence of entanglements between tethered chains. The similarity of the effect of polymer-graft 
modification on the interaction between lignin fillers to those observed in inorganic particle brush analogs motivates the hypothesis that polymer-graft modification should be an effective method to facilitate the improved dispersion of lignin in polymer matrices and the fabrication of bio-sourced polymer composites with enhanced mechanical properties. $(18,19)$

The purpose of this contribution is to illustrate the application of SI-ATRP to the synthesis of well-defined poly(methyl methacrylate) (PMMA)-tethered kraft lignin and its application as toughening agent in PMMA-based nanocomposites. It is demonstrated that the tethering of polymeric chains renders kraft lignin a highly effective additive that is capable of raising both the stiffness and toughness of PMMA at filler concentrations as low as $0.5 \mathrm{wt} \%$. The resulting biomass-derived nanocomposites exhibit property characteristics that rival high performance engineering plastics and point to new opportunities for the application of bio-sourced fillers by harnessing advanced surface polymerization techniques.

The material system in our study consists of PMMA-tethered kraft-lignin particles dispersed in a PMMA matrix with $N_{\mathrm{m}}=680$, where $N_{\mathrm{m}}$ denotes the degree of polymerization of the matrix polymer. PMMA was chosen as model system because of its important role as transparent polymer glass with a global market that is expected to exceed $\$ 9.7$ billion per year by 2017. Enhancing the mechanical properties of PMMA while reducing the energy and environmental footprint of the material is of significant technological importance since pristine PMMA - due to its amorphous character exhibits rather limited modulus and toughness that often constrain its application. 


\section{Experimental Section}

\subsection{Materials}

Lignin was purchased from TCI America. Dimethylformamide (DMF), methyl methacrylate (MMA), azobisisobutyronitrile (AIBN), bromoisopropionyl bromide, $\mathrm{CuBr}$, $\mathrm{N}, \mathrm{N}, \mathrm{N}^{\prime}, \mathrm{N}^{\prime}, \mathrm{N}^{\prime \prime}$-pentamethyldiethylenetriamine (PMDETA), and pentafluorobenzaldehyde were purchased from Sigma-Aldrich, Inc. Lignin was acidified using previously reported methods,(15) and MMA monomer was passed through a basic alumina column before use to remove inhibitor. All other chemicals were used as received.

\subsection{Radical Polymerization and Sample Preparation}

Methyl methacrylate was polymerized in toluene using an AIBN initiator to prepare the PMMA homopolymer used in this work with an $\mathrm{M}_{\mathrm{n}}$ of $68,000 \mathrm{~g} / \mathrm{mol}$ and a polydispersity ratio of 2.35. ATRP was used to prepare PMMA-grafted lignin using previously reported methods.(15) A lignin macroinitiator was prepared by reacting acidified lignin with $\alpha$-bromoisobutyryl bromide in DMF. For MMA polymerization from the lignin macroinitiator, $0.1915 \mathrm{~g}$ initiator (ca. $0.126 \mathrm{mmol}), 18.8 \mathrm{mg} \mathrm{CuBr}(0.131 \mathrm{mmol})$, and 26.3 mL PMDETA $(0.126 \mathrm{mmol})$ were added to the Schlenk flask and degassed using three vacuum-nitrogen cycles. DMF and MMA were bubbled under nitrogen for 30 min then DMF $(7.5 \mathrm{~mL})$ and MMA $(2.63 \mathrm{~mL}, 24.6 \mathrm{mmol})$ were added to the flask. The polymerization was carried out at $70^{\circ} \mathrm{C}$ for $24 \mathrm{~h}$. 


\subsection{Nanocomposite Preparation}

Solvent blending in minimal amounts of pyridine was used to prepare filled PMMA. The samples were pressed into sheets and vacuum annealed at $180^{\circ} \mathrm{C}$ for 1 day then stored at room temperature prior to use.

\subsection{Characterization}

\section{NMR}

NMR measurements were carried out using a Bruker Advance $300 \mathrm{MHz}$ NMR Instrument in $\mathrm{CDCl}_{3}$ or deuterated dimethyl sulfoxide. The number of initiator sites per mass of lignin was determined with $10 \mu \mathrm{L}$ of pentafluorobenzaldehyde as an internal standard. $\left[{ }^{1} \mathrm{H}\right.$ NMR (300 MHz): $\delta=2.0 \mathrm{ppm}(\mathrm{s}, 6 \mathrm{H}$, conjugated initiator), $\delta=10.19 \mathrm{ppm}(\mathrm{s}$, $1 \mathrm{H}, \mathrm{PFB})$.

\section{Dynamic Light Scattering}

Size distributions of particles were measured in a pyridine at a concentration of 1 $\mathrm{mg} / \mathrm{mL}$. The solution was ultrasonicated for 2 minutes prior to being placed in the glass cuvette. The particle diameter was measured using a Zeta-sizer DLS (Malvern Instruments).

\section{Tensile Testing}

Tensile stress-strain tests were performed on an Instron tensile test machine using pneumatic grips and operated at a crosshead speed of $0.0166 \mathrm{~mm} / \mathrm{sec}$. Specimen for 
tensile test evaluation were prepared by first hot-pressing of samples at $120{ }^{\circ} \mathrm{C}$ and subsequent cutting of strips with test region dimensions of $20 \mathrm{~mm} \times 5 \mathrm{~mm} \times 1.5 \mathrm{~mm}$. The experimental error was estimated by evaluating the error associated with the measurement of five test specimens.

\section{Differential Scanning Calorimetry}

Thermal properties of the filled polymers were measured by DSC Q20 (TA Instruments) using a heating rate of $5{ }^{\circ} \mathrm{C} / \mathrm{min}$. Samples were run in triplicate to determine the error in the measurements.

\section{Transmission Electron Microscopy}

For transmission electron microscopy, the PMMA samples were microtomed at $-120{ }^{\circ} \mathrm{C}$ to a section thickness of $70 \mathrm{~nm}$ and stained with $\mathrm{OsO}_{4}$ for 20 minutes. All samples were imaged using JEOL 2000EX transmission electron microscope operated at $200 \mathrm{keV}$ in bright field mode.

\section{Results and Discussion}

Surface-initiated controlled radical polymerization provides a facile and effective strategy for the functionalization of nanoparticles and biopolymers with a wide range of polymer graft compositions.(13) A general advantage of this 'grafting-from' approach is the fine control of relevant molecular parameters such as the grafting density as well as the potential applicability to more complex compositions such as bimodal polymer grafts.(14) Kadla and coworkers were the first to demonstrate the application of ATRP to 
the synthesis of polymer-modified lignin derivatives. In particular the authors grafted Nisopropylacrylamide from kraft lignin to impart temperature-dependent solubility.(20) In this work it is demonstrated that the application of SI-ATRP for the modification of lignin with high-molecular (i.e. the molecular weight exceeds the threshold value for entanglement formation) PMMA along with its application for the preparation of PMMA/lignin composites with enhanced mechanical properties.

Polymer-graft modification using SI-ATRP followed a two-step procedure: In a first step the initiator (here: bromoisobutyryl bromide) was coupled to hydroxyl functionalities of kraft lignin and subsequently was used to polymerize MMA via ATRP as described previously.(15) In Scheme 1 is shown the synthetic strategy for preparing PMMA-grafted lignin using ATRP.

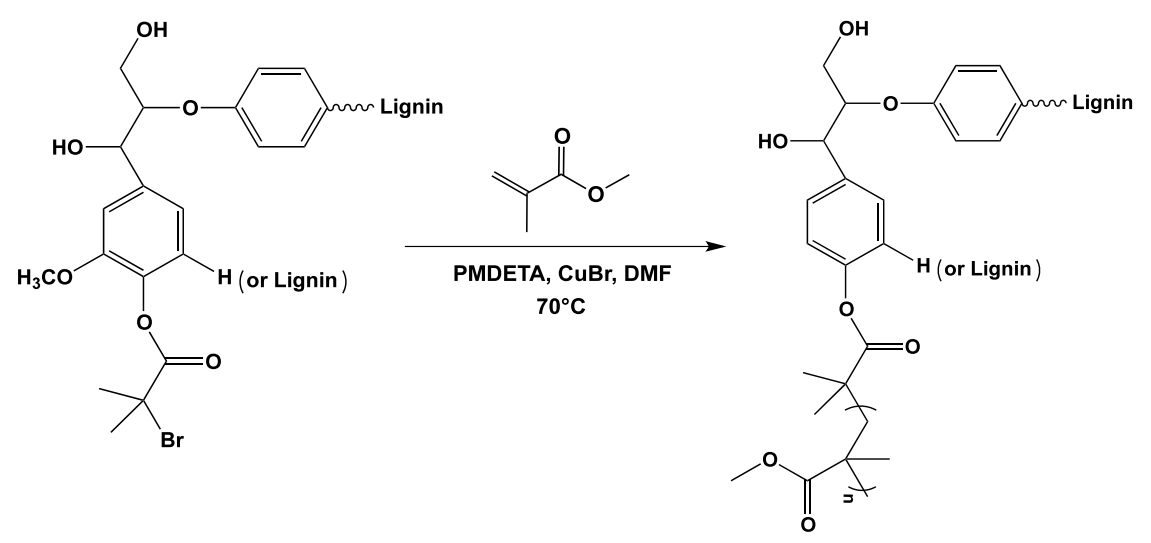

Scheme 1. Methyl methacrylate polymerization from a lignin-based ATRP initiator.

NMR and GPC analysis revealed a grafting density of $<0.1 / \mathrm{nm}^{2}$ and a degree of polymerization of $N_{\mathrm{G}}=388$ of surface tethered chains. Based on an estimated average lignin molecular mass of $25 \mathrm{kDa}$, each lignin particle carries approximately 8 PMMA 
grafts with a standard deviation of about $30 \%$ in the number of grafts per particle. $(21,22)$ It is estimated that approximately $15 \%$ of lignin hydroxyl groups were functionalized with ATRP initiators using these conditions. The degree of polymerization was chosen because it significantly exceeds the critical entanglement segment length of PMMA $\left(N_{\mathrm{c}} \sim\right.$ $330)(23)$ while retaining appreciable lignin character of the hybrid particle $\left(\phi_{\text {lignin }} \sim 0.12\right)$. Polymer ligands in surface-grafted particle systems have been shown to exhibit a range of conformational states (with segments assuming more stretched conformations in the vicinity of the particle surface and more relaxed conformations at larger distance) depending on the interaction of surface grafted chains. If only excluded volume interactions are considered than chain interactions are expected to depend on three primary parameters, i.e. the particle size (or surface curvature), the grafting density and the degree of polymerization of surface-bound chains. Kumar and coworkers(10) demonstrated that the chain conformation of surface-grafted chains can be approximately evaluated based on a scaling model that was first introduced by Daoud-Cotton to describe chain conformational transitions in star polymers and later extended by Ohno et al. to polymer-tethered particle systems $(18,24,25)$. Under the assumption of lignin acting like a 'organic particle' with a geometric radius of $R \cong 2.5 \mathrm{~nm}$ (consistent with DLS measurements), the application of this model suggests that PMMA ligands assume a relaxed chain conformation.(26) In the following, lignin fillers will be denoted as 'lignin$g$ MMA $N$ ' where $g$ denotes the average number of polymer grafts per lignin and $N$ the average degree of polymerization of grafted chains. 
The effectiveness of PMMA-modification in enhancing the solubility of kraft lignin as well as the size of dispersed lignin particles was determined using dynamic light scattering (DLS) that revealed uniform distribution of particle size with an average hydrodynamic particle diameter of $5 \mathrm{~nm}$ and $28 \mathrm{~nm}$ for pristine and PMMA-grafted lignin in dilute DMF solution, respectively, as shown in Figure 1a. In the following 28 $\mathrm{nm}$ will be assumed to be the characteristic length scale of lignin-8MMA388 filler particles (although some aggregation might be inferred from the width of the distribution of hydrodynamic radii). The 'particulate nature' of lignin-8MMA388 is confirmed by TEM such as shown in Figure $1 \mathrm{~b}$ that reveals an average particle size of $<\mathrm{d}>\sim 54 \pm 16$ nm. It is hypothesized that 'particle stacking' in the case of the rather dense lignin8MMA388 films could cause an overestimate of the apparent lignin-particle size. For this reason and because of the more representative analysis of light scattering it will be considered the hydrodynamic size as a reference measure for the size of individual particle fillers.

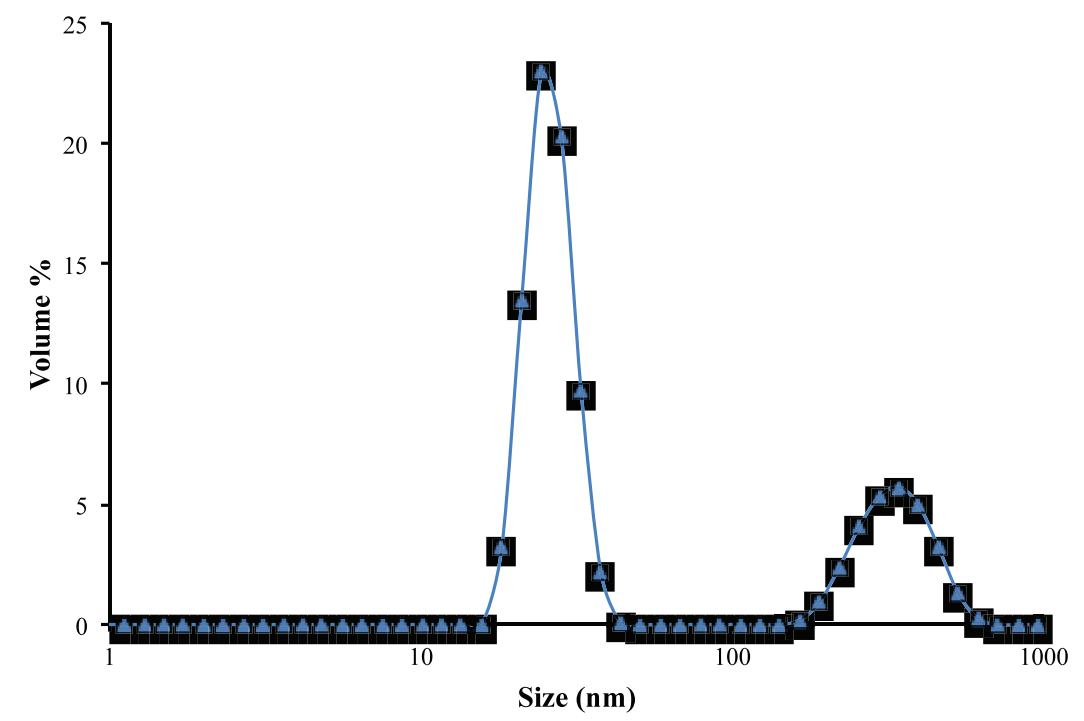




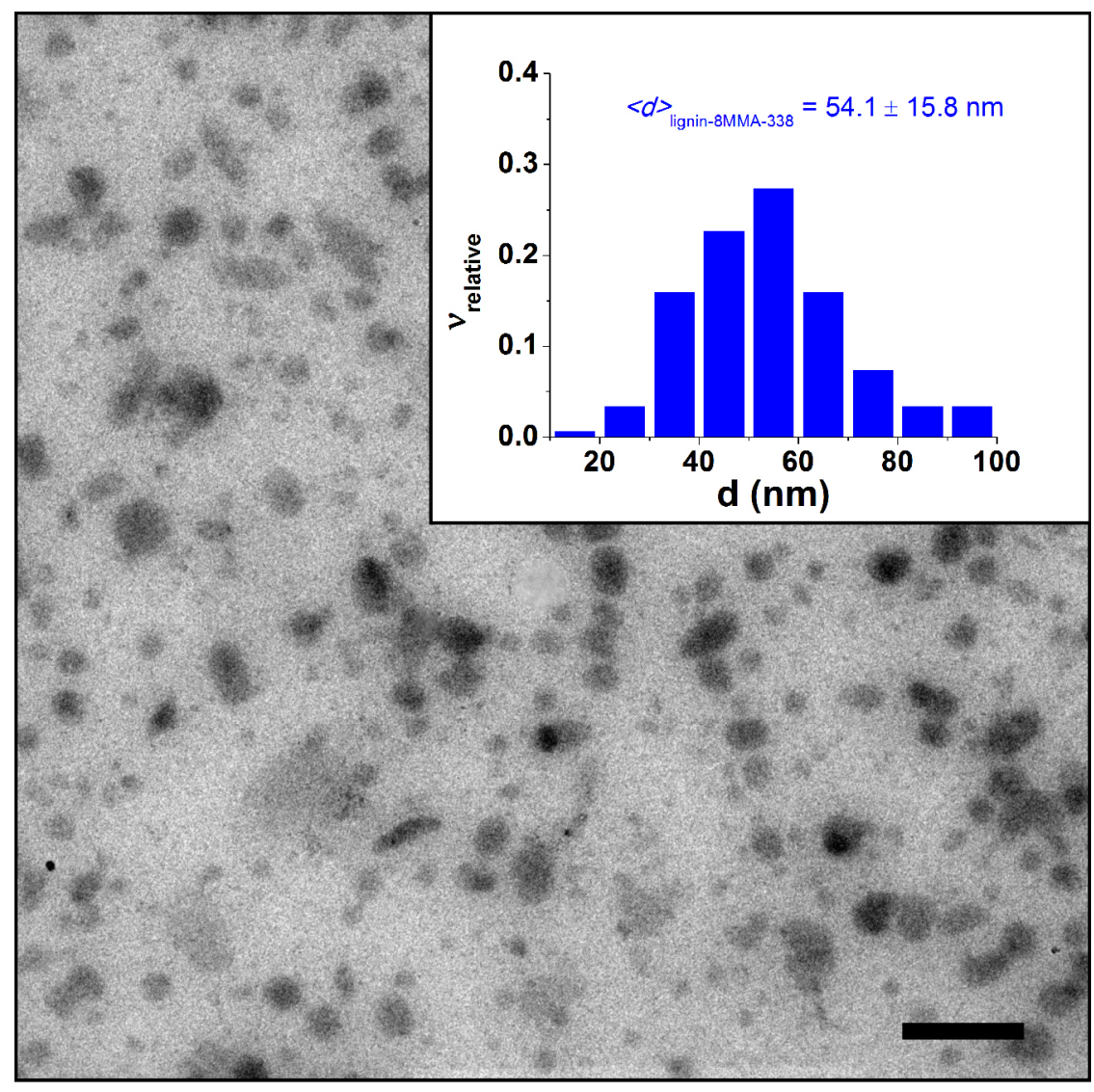

Figure 1. (a) DLS characterization of lignin-8MMA388 in pyridine. The size of the peak centered around $250 \mathrm{~nm}$ is concentration dependent and attributed to aggregation. (b) Transmission electron micrograph of lignin-8MMA388 cast from toluene solution. Scale bar is $200 \mathrm{~nm}$. Inset shows particle size distribution.

The thermo-mechanical properties of pristine lignin-8MMA388 were determined using tensile and calorimetric characterization (see 'Experimental Section' and Hilburg et al.(15)) are provided in Table 1 as reference for the following discussion. 
Table 1. Summary of material properties of lignin-8MMA388.

PMMA/lignin-8MMA388 nanocomposites with $0.5,1$ and $2 \%$ filler content were prepared by solution casting and subsequent annealing under vacuum for 1 day at $180{ }^{\circ} \mathrm{C}$. Composites were found to remain optically transparent although a faint amber coloration was observed with increasing lignin content (not shown here).

Figure 2 depicts a representative transmission electron micrograph revealing the microstructure of the PMMA/lignin-8MMA388 composite after 1 day of thermal annealing ( $1 \mathrm{wt} \%$ filler content; the lignin component was preferentially stained with $\mathrm{OsO}_{4}$ ). The micrograph reveals individual dispersion of lignin fillers with an average particle size that is determined from large area analysis (not shown here) to be $<\mathrm{d}>\sim 19 \pm$ $5 \mathrm{~nm}$ - a value that is in good agreement with the corresponding hydrodynamic size of lignin-8MMA388 fillers (the corresponding size distribution of aggregate structures is shown in the inset of Fig. 2). 


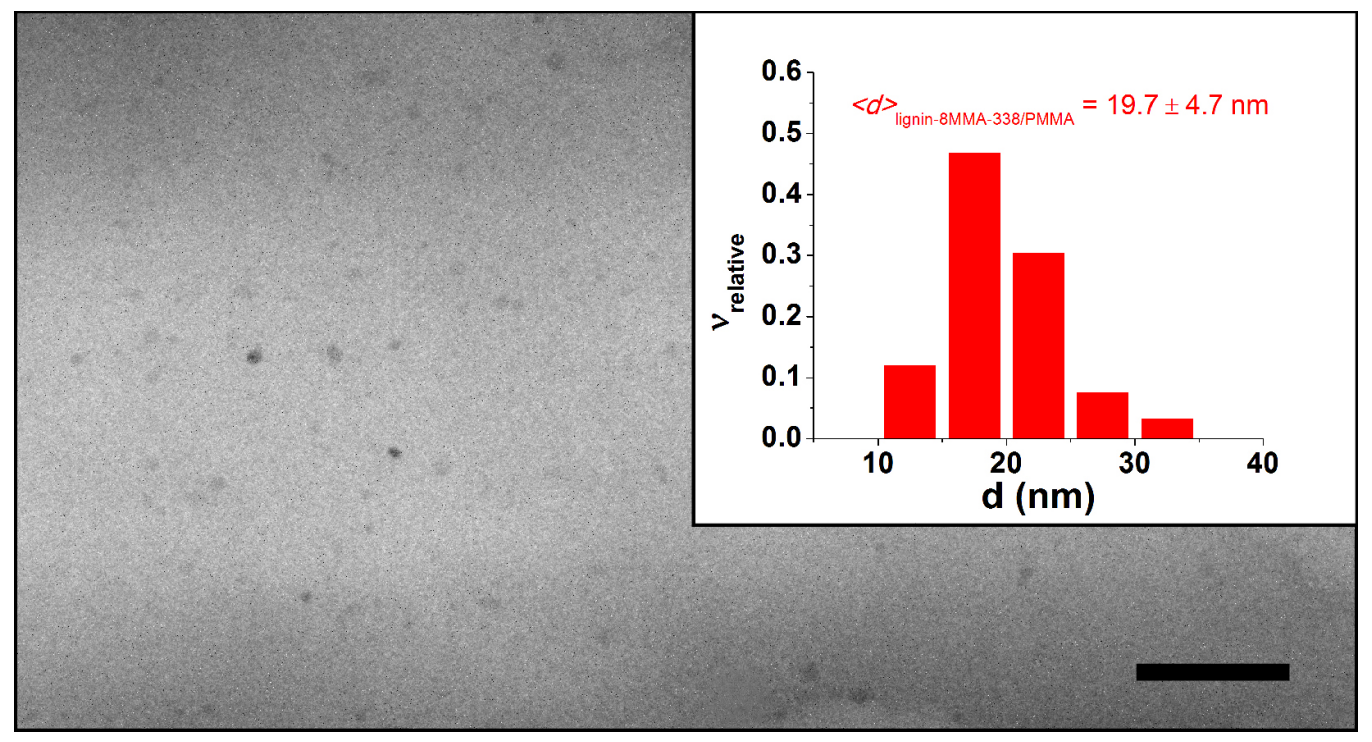

Figure 2. Representative transmission electron micrograph depicting the microstructure of PMMA/lignin-8MMA388 (1 wt\%). Scale bar is $200 \mathrm{~nm}$. Inset shows particle size distribution.

It is noted that the good particle dispersion is in contrast to previous reports on lignin composites in which aggregation and phase separation has been observed.(7) It is thus concluded that PMMA-grafting does indeed enhance the compatibility of lignin nanofillers within the PMMA matrix.

Figure 3 displays the representative stress-strain data for PMMA/lignin-8MMA388 composites revealing a significant improvement of the fracture toughness in composite systems. Interestingly, while the $2 \%$ samples does show significant softening (i.e. a reduction of Young's modulus by 40\%) the PMMA/lignin-8MMA388 system reveals both increased modulus and fracture toughness at filler content $\leq 1 \mathrm{wt} \%$. This trend was stable among 6 test samples for each composition that were evaluated to ascertain the 
reproducibility of results (indicated by the error bars in Figure 4). In particular, the Young's modulus, ultimate tensile strength, and ultimate elongation for the $1 \%$ lignin-gPMMA system were $659 \pm 10 \mathrm{MPa}, 23.0 \pm 10 \mathrm{MPa}$, and $0.12 \pm 0.02 \mathrm{MPa}$; as compared to $429 \pm 20 \mathrm{MPa}, 6.9 \pm 2 \mathrm{MPa}$, and $0.025 \pm 0.003$ for pristine PMMA, respectively.

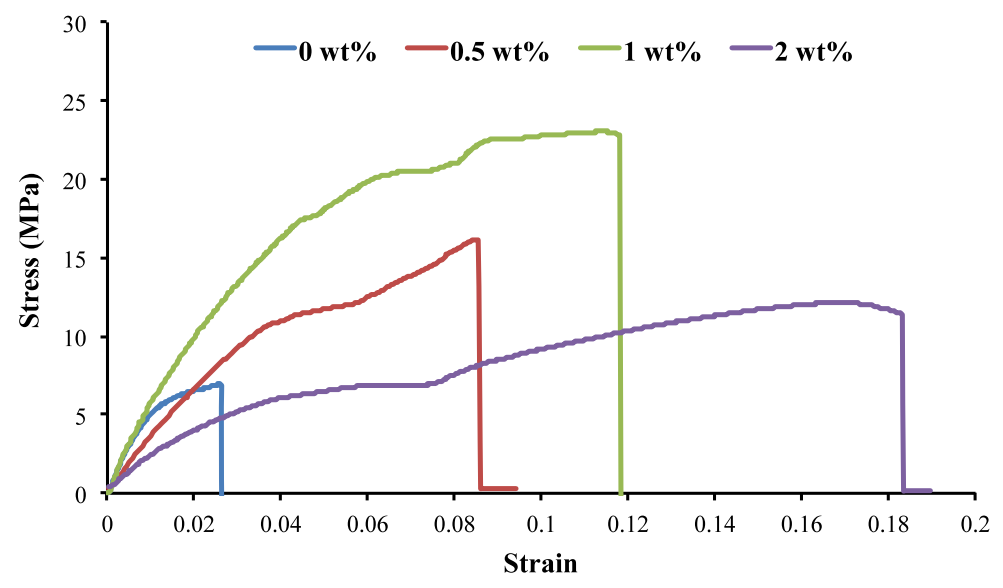

Figure 3. Representative engineering stress versus strain data for pristine PMMA (blue) and PMMA/lignin-8MMA388 composites with filler concentration $0.5 \mathrm{wt} \%$ (red), $1 \mathrm{wt} \%$ (green) and $2 \mathrm{wt} \%$ (purple).

The increase in Young's modulus provides direct evidence for improved interfacial bonding between filler and matrix that is facilitated by polymeric tethers. One particularly interesting aspect of the effect of lignin addition is the increase of the materials' tensile toughness that is determined by integration of the stress $v s$ strain data. In case of PMMA/lignin-8MMA388 (1\%) system an increase by about $800 \%$ as compared to pristine PMMA is observed thus rendering the material more comparable with high performance engineering polymers. The favorable effect of lignin addition is highest at $1 \mathrm{wt} \%$ filler content while at 2\% lignin-g-PMMA, significant plasticization 
was observed with corresponding decreases in Young's modulus and tensile strength and increase in strain at fracture. It is hypothesized that the softening effect is caused by the small interparticle distance at $2 \mathrm{wt} \%$ filler concentration that could significantly alter the properties of the polymer matrix as well as filler matrix interactions. In particular, the interparticle distance can be estimated as $(N / V)^{-1 / 3}=46,35$, and $28 \mathrm{~nm}$ for $0.5,1$, and 2 wt $\%$ filler concentration, respectively ( $N / V$ denotes the lignin particle number density). Hence, the distance between particle fillers in the PMMA/lignin-8MMA388 (2\%) system approaches the characteristic dimension of the matrix polymer (see also discussion below).

The trends in mechanical properties as a function of loading level are summarized in Figure 4. 

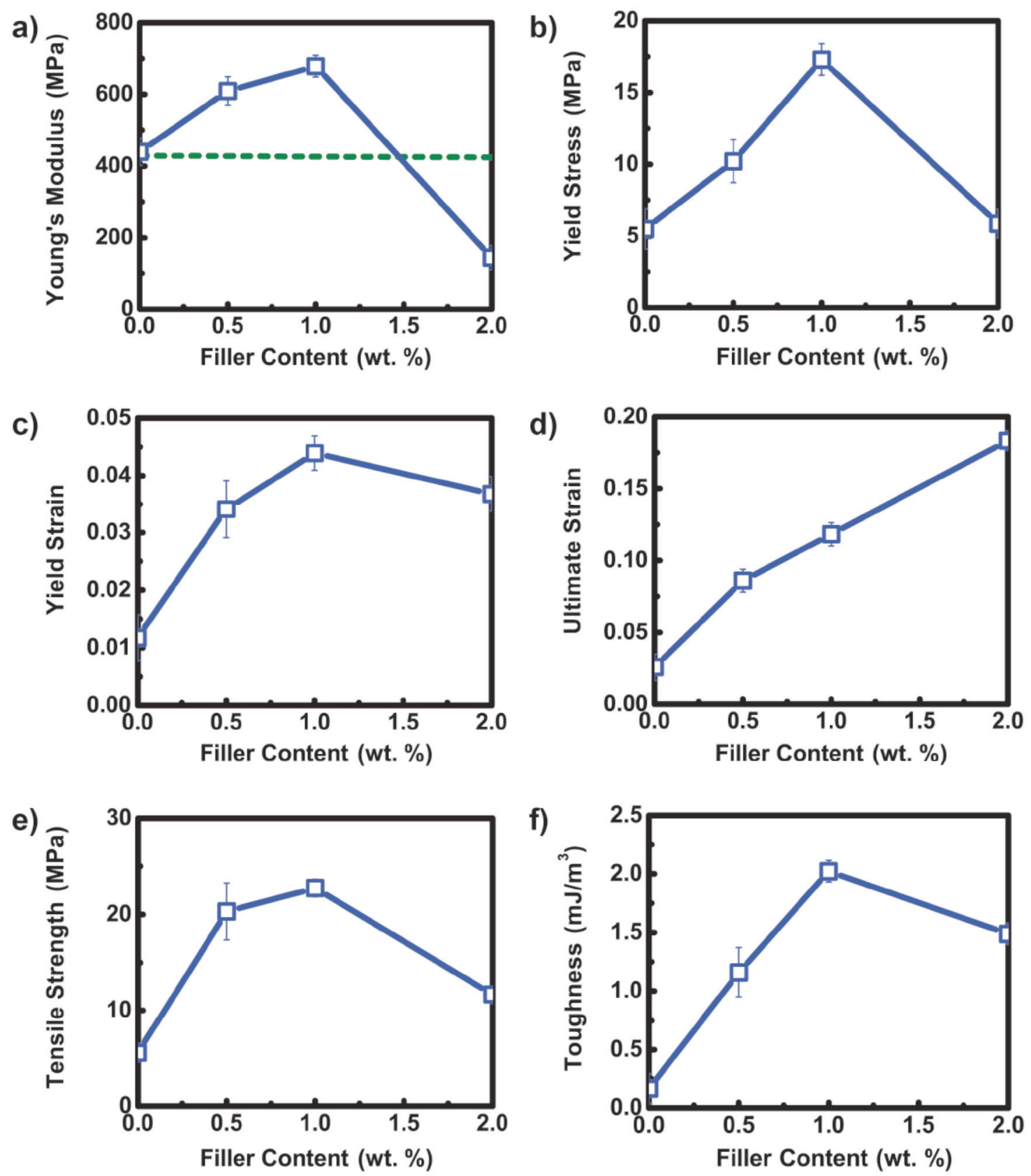
Figure 4. Summary of mechanical properties of PMMA/lignin-8MMA388 composite systems determined from tensile stress-strain data shown in Figure 3: (a) Young's modulus; (b) yield stress; (c) yield strain; (d) strain-at-fracture; (e) ultimate tensile strength; (f) tensile toughness. The rule-of-mixtures prediction for the Young's modulus in (a) is represented as a dashed line.

It is interesting to note that the observed increase in elastic modulus significantly exceeds the upper bound that is predicted on the basis of homogenization theory that is indicated as the dotted line in Figure 4a [footnote: The upper bound for the effective modulus of the PMMA/lignin-8MMA388 composite was determined using the rule-of-mixtures as $E_{\mathrm{eff}}=E_{\mathrm{PMMA}} \phi_{\mathrm{PMMA}}+E_{\text {lignin-8MMA388 }} \phi_{\text {lignin-8MMA388, }}$, see reference(3)]. The enhancement effect that is observed in the presence of small amounts of lignin fillers bears resemblance to reported strengthening effects in the case of inorganic fillers.(27) In reference to these previous observations the enhancement is interpreted to be a consequence of the interfacial bonding between filler and matrix that is facilitated by the presence of polymer grafts and possibly strong interactions between the lignin core and the PMMA matrix. It is hypothesized that the effect is related to the temperature dependence of the directional bonds that could exist in PMMA/lignin systems (due to the presence of hydroxyl functionalities in lignin). Since directional bond interactions become unstable above a threshold temperature (typically of the order of $60-70{ }^{\circ} \mathrm{C}$ ) their 
contribution to the low-temperature elastic modulus and glass temperature could be more complex.

To gain further insight into the role of filler addition on the structure formation and interactions in polymer/lignin blend systems thermal transitions were analyzed using differential scanning calorimetry. Figure 5 shows the effect of lignin-8MMA388 addition on the glass temperature of the composite, revealing a weak decrease of $T_{\mathrm{g}}$ at low filler concentrations and a pronounced drop (by $T_{\mathrm{g}} \sim 22{ }^{\circ} \mathrm{C}$ ) at $2 \mathrm{wt} \%$ filler content. The pronounced reduction of $T_{\mathrm{g}}$ is consistent with the softening behavior that is observed at higher filling fraction and bears similarity to the softening behavior in silica/PMMA composites that has been attributed to 'spatial confinement' of matrix chains. Spatial confinement relates to the energetic frustration that is experienced by matrix chains when the average distance between filler particles is less than the equilibrium chain end-to-end distance. Here, it is noted that the average particle distance at $2 \mathrm{wt} \%$ filler content is approximately equal to the end-to-end distance of the matrix chain $\left(R_{\mathrm{e}} \sim 40 \mathrm{~nm}\right)$. It is hypothesized that spatial confinement results in softening of the matrix that counteracts the strengthening effect of the matrix - but more research will have to be performed to elucidate the experimental trend.

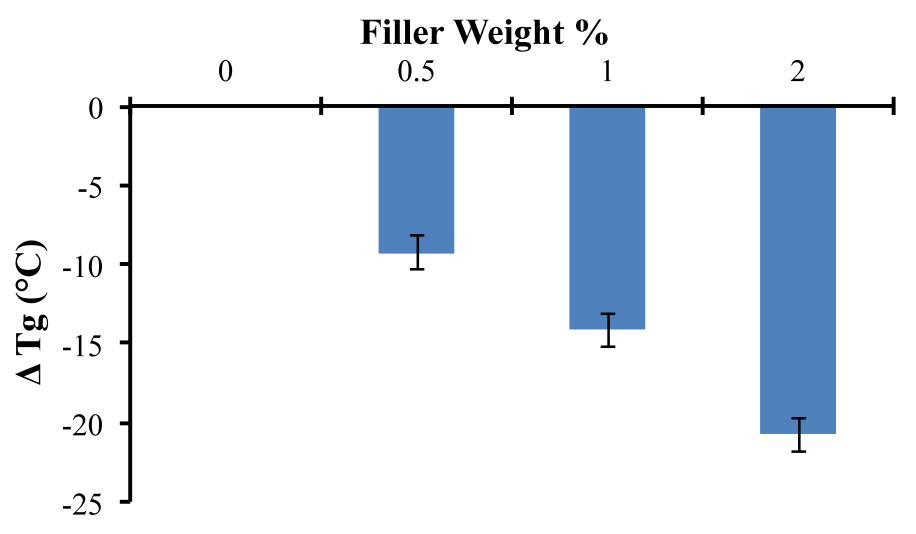


Figure 5. Difference in $T_{g}$ as a function of filler loading level relative to unfilled PMMA.

\section{Conclusions}

In summary, it is demonstrate that surface-initiated atom transfer radical polymerization facilitates the tethering of polymer chains with sufficiently high degree of polymerization to facilitate entanglements between surface-grafted chains. For the case of PMMAtethered graft lignin dispersed in PMMA polymer-graft modification is shown to dramatically enhance the mechanical properties of the matrix, thus rendering its properties more similar to high-performance materials such as polycarbonate. The existence of an 'optimum filler concentration' for property enhancement is observed that is interpreted as a consequence of the competing influence of filler-induced reinforcement, and stress concentration around larger particle aggregates. Optimum property enhancements including a 3-fold increase in yield stress, a 4-fold increase in tensile strength, and a 7-fold increase in toughness are realized at minute filler content of $1 \%$. The dramatic property enhancement in case of PMMA at very low filler concentration (at which optical transparency is retained) points to new opportunities of bio-sourced fillers (such as graft-lignin) as additives in high performance commoditybased polymers that could provide more benign alternatives to more expensive specialty engineering polymers or nanocomposites based on inorganic nanoparticles. Future work will focus on the role of the degree of polymerization as well as grafting density of 
surface tethered chains that are expected to be critical governing parameters for the dispersion as well as interaction in lignin/polymer composite systems.

Keywords: lignin; ATRP; nanocomposite; renewable materials; plastics

\section{Acknowledgments}

MRB acknowledges support by the National Science Foundation (via grant DMR-

1006473 and DMR-1410845). NMR instrumentation at CMU was partially supported by NSF (CHE-0130903 and CHE-1039870).

\section{References}

1. Caseri WR. Nanocomposites of polymers and inorganic particles: preparation, structure and properties. Mater Sci Tech-Lond. 2006;22(7):807-17.

2. Dang A, Ojha S, Hui CM, Mahoney C, Matyjaszewski K, Bockstaller MR. Hightransparency polymer nanocomposites enabled by polymer-graft modification of particle fillers. Langmuir. 2014;30(48):14434-42.

3. Krishnamoorti R, Vaia RA. 2002. American Chemical Society, Division of Polymeric Materials: Science and Engineering. "Polymer nanocomposites : synthesis, characterization, and modeling."

4. Winey KI, Vaia RA. Polymer nanocomposites. MRS Bull. 2007;32(4):314-9.

5. Chung H, Washburn NR. Chemistry of lignin-based materials. Green Mater. 2012;1(3):137-60.

6. Kadla JF, Kubo S. Lignin-based polymer blends: analysis of intermolecular interactions in lignin-synthetic polymer blends. Compos Part a-Appl S. 2004;35(3):395400 . 
7. Ciemniecki SL, Glasser WG. Multiphase Materials with Lignin .1. Blends of Hydroxypropyl Lignin with Poly(Methyl Methacrylate). Polymer. 1988;29(6):1021-9. 8. Hui CM, Pietrasik J, Schmitt M, Mahoney C, Choi J, Bockstaller MR, et al. Surface-Initiated Polymerization as an Enabling Tool for Multifunctional (Nano)Engineered Hybrid Materials. Chem Mater. 2014;26(1):745-62.

9. Akcora P, Liu H, Kumar SK, Moll J, Li Y, Benicewicz BC, et al. Anisotropic self-assembly of spherical polymer-grafted nanoparticles. Nat Mater. 2009;8(4):354U121.

10. Harton SE, Kumar SK. Mean-field theoretical analysis of brush-coated nanoparticle dispersion in polymer matrices. J Polym Sci Pol Phys. 2008;46(4):351-8. 11. Kumar SK, Jouault N, Benicewicz B, Neely T. Nanocomposites with Polymer Grafted Nanoparticles. Macromolecules. 2013;46(9):3199-214.

12. Ojha S, Dang A, Hui CM, Mahoney C, Matyjaszewski K, Bockstaller MR. Strategies for the synthesis of thermoplastic polymer nanocomposite materials with high inorganic filling fraction. Langmuir. 2013;29(28):8989-96.

13. Barbey R, Lavanant L, Paripovic D, Schuwer N, Sugnaux C, Tugulu S, et al. Polymer Brushes via Surface-Initiated Controlled Radical Polymerization: Synthesis, Characterization, Properties, and Applications. Chem Rev. 2009;109(11):5437-527.

14. Rungta A, Natarajan B, Neely T, Dukes D, Schadler LS, Benicewicz BC. Grafting Bimodal Polymer Brushes on Nanoparticles Using Controlled Radical Polymerization. Macromolecules. 2012;45(23):9303-11.

15. Hilburg SL, Elder AN, Chung H, Ferebee R, Bockstaller MR, Washburn NR. A Universal Route Towards Thermoplastic Lignin Composites with Improved Mechanical Properties. Polymer. 2014;55(4):995-1003.

16. Matyjaszewski K, Tsarevsky NV. Nanostructured functional materials prepared by atom transfer radical polymerization. Nat Chem. 2009;1(4):276-88.

17. Wang JS, Matyjaszewski K. Controlled Living Radical Polymerization - AtomTransfer Radical Polymerization in the Presence of Transition-Metal Complexes. J Am Chem Soc. 1995;117(20):5614-5.

18. Choi J, Dong H, Matyjaszewski K, Bockstaller MR. Flexible Particle Array Structures by Controlling Polymer Graft Architecture. J Am Chem Soc. 2010;132(36):12537-9.

19. Tchoul MN, Fillery SP, Koerner H, Drummy LF, Oyerokun FT, Mirau PA, et al. Assemblies of Titanium Dioxide-Polystyrene Hybrid Nanoparticles for Dielectric Applications. Chem Mater. 2010;22(5):1749-59.

20. Kim YS, Kadla JF. Preparation of a Thermoresponsive Lignin-Based Biomaterial through Atom Transfer Radical Polymerization. Biomacromolecules. 2010;11(4):981-8.

21. Hakem IF, Leech AM, Bohn J, Walker JP, Bockstaller MR. Analysis of heterogeneity in nonspecific PEGylation reactions of biomolecules. Biopolymers. 2013;99(7):427-35.

22. Hakem IF, Leech AM, Johnson JD, Donahue SJ, Walker JP, Bockstaller MR. Understanding Ligand Distributions in Modified Particle and Particlelike Systems. J Am Chem Soc. 2010;132(46):16593-8.

23. Fetters LJ, Lohse DJ, Graessley WW. Chain dimensions and entanglement spacings in dense macromolecular systems. J Polym Sci Pol Phys. 1999;37(10):1023-33. 
24. Daoud M, Cotton JP. Star Shaped Polymers - a Model for the Conformation and Its Concentration-Dependence. J Phys-Paris. 1982;43(3):531-8.

25. Ohno K, Morinaga T, Takeno S, Tsujii Y, Fukuda T. Suspensions of silica particles grafted with concentrated polymer brush: Effects of graft chain length on brush layer thickness and colloidal crystallization. Macromolecules. 2007;40(25):9143-50. 26. Choi J, Hui CM, Pietrasik J, Dong HC, Matyjaszewski K, Bockstaller MR. Toughening fragile matter: mechanical properties of particle solids assembled from polymer-grafted hybrid particles synthesized by ATRP. Soft Matter. 2012;8(15):4072-82. 27. Ash BJ, Rogers DF, Wiegand CJ, Schadler LS, Siegel RW, Benicewicz BC, et al. Mechanical properties of A12O3/polymethylmethacrylate nanocomposites. Polym Composite. 2002;23(6):1014-25. 

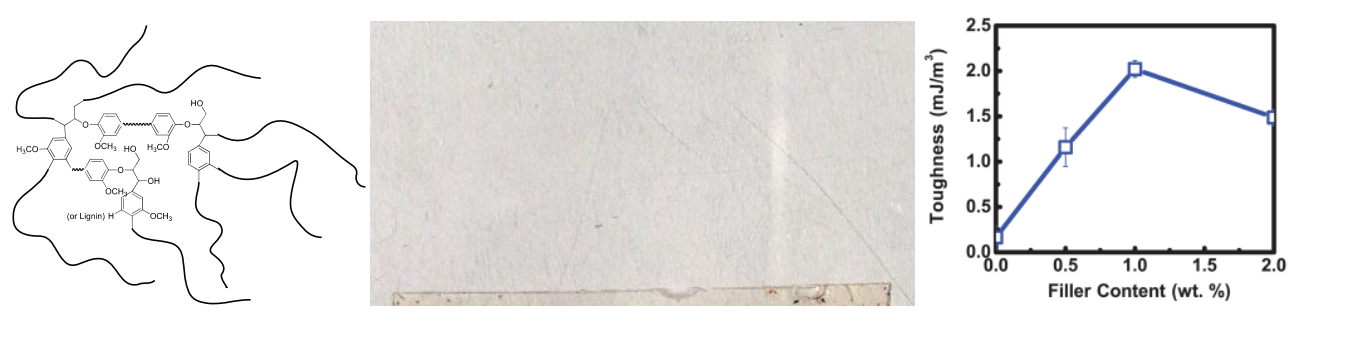

*Graphical Abstract

(F) 